\title{
Anatomic Variation in Palatal Root of Maxillary Second Molar: Endodontic Management
}

\author{
Juan G. Robledo, Pablo A. Rodriguez \\ Endodontics Department, School of Dentistry, University of Buenos Aires, Buenos Aires, Argentina \\ Email: juan.robledo@odontologia.uba.ar,pablorodriguez@dentalmedicine.com.ar
}

How to cite this paper: Robledo, J.G. and Rodriguez, P.A. (2021) Anatomic Variation in Palatal Root of Maxillary Second Molar: Endodontic Management. Open Journal of Stomatology, 11, 159-165.

https://doi.org/ 10.4236/ojst.2021.114013

Received: January 4, 2021

Accepted: April 3, 2021

Published: April 6, 2021

Copyright (อ 2021 by author(s) and Scientific Research Publishing Inc. This work is licensed under the Creative Commons Attribution International License (CC BY 4.0).

http://creativecommons.org/licenses/by/4.0/

\begin{abstract}
The aim of the present clinical case report is to describe the singular root canal anatomy and endodontic management of a maxillary second molar with two palatal roots and two separate canal orifices. A 60 years-old female Hispanic/Latino patient attended the Endodontic Department at University of Buenos Aires School of Dentistry with acute pain in her maxillary left second molar. Clinical radiographs examination revealed unusual root morphology. Cleaning and shaping were completed with $5.25 \% \mathrm{NaOCl}$ irrigation, $15 \%$ EDTA solution and hand/rotary instruments. Filling was performed with gutta-percha points and resin-based sealer. After one year follow-up periapical radiographs and CBCT images showed healing in the area and patient was asymptomatic.
\end{abstract}

\section{Keywords}

Aberration, Anatomic Variation, Maxillary Second Molar, Two Palatal Roots

\section{Introduction}

Presence of bacteria in the root canal system is a crucial dilemma in endodontics. This may affect the endodontic outcome stimulating periradicular tissue inflammation, reinforcing the theory that elimination of bacteria should be the most important goal in endodontics therapy [1]. Incomplete elimination of etiologic factors, irritations and contamination of the root canal system are the main causes for failure of nonsurgical and surgical root canal therapy [2]. Generally, maxillary second molar has been described to have three roots and three or four canals, with the fourth canal located in the mesiobuccal root. Some authors reported when an extra palatal root is detected, one is the normal palatal root and the other is a supernumerary structure. The etiology remains still ambiguous, this anatomic variation could be associated to the splitting of the Hertwig's epi- 
thelial root sheath by exogenic or endogenic factors during the root development process [3]. However, other authors expressed that the origin could be racial genetic factors and/or external factors during odontogenesis [4].

Second maxillary molars with two palatal roots are rare. Peikoff et al. [5] observed 520 root canal treatments of maxillary second molar teeth and found 1.4\% of maxillary second molars presenting two palatal roots. Libfeld and Rotstein [6] carried out a study of 1200 in order to establish the occurrence of four-rooted maxillary second molars. Teeth were examined radiographically and the outcome evidenced that only $0.4 \%$ of the teeth showed with four separate roots. In addition, there were some clinical case reports of maxillary second molars evidencing the presence of two palatal roots [7] [8].

Christie et al. [9] presented a classification in three types of maxillary teeth with two palatal root canals: Type I have two widely divergent palatal roots. The buccal roots of these teeth are often "cow-horn" shaped and less divergent. Type II has four separate roots also but the roots are often shorter; Type III with the mesiobuccal, mesiopalatal, and distopalatal canal confined in a web of root dentin and the distobuccal root in these cases appears to stand alone. The aim of the present clinical case report is to show the uncommon root canal anatomy of a Type II maxillary molar with two separated palatal roots and the endodontic management for this tooth.

\section{Case Report}

A 60-year-old Hispanic/Latino female attended the Endodontics Department at University of Buenos Aires because of severe pain in her left maxillary second molar. The discomfort was increased by percussion and thermal stimulation. Medical history was non-relevant and the patient refused any systemic disease and allergy. Clinical and radiographic examination showed that tooth had an amalgam on the distal part of the crown and a temporary restoration extended into the pulp chamber that was placed 10 days previously (Figure 1(a) and Figure 1(b)). Periodontal examination was registered within normal limits. After evaluation of clinical and radiographic findings the tooth was associated with symptomatic apical periodontitis.

Local infiltration anesthesia was administered using 4\% carticaine and 1:100.000 adrenaline (Anescart Forte, SIDUS, Buenos Aires, Argentina) followed by dental dam isolation. After temporary filling removal, the access cavity was prepared using round burs. The pulp chamber was then irrigated with $5.25 \%$ of sodium hypoclorite $(\mathrm{NaOCl})$ and the orifice canals were located under operating microscope (Newton SRL, Buenos Aires, Argentina) using a DG16 endodontic explorer (Hu-Friedy, Chicago, IL, USA). Size 10 stainless steel K-files (Dentsply, Ballaigues, Switzerland) were used to establish an initial glidepath to the desired apical location. The working length was verified using an electronic apex locator (Root ZX, J.Morita, Kyoto, Japan) and confirmed radiographically (Figure 1(c)).

Under copious irrigation with $5.25 \% \mathrm{NaOCl}$, the four canals $(\mathrm{MB}, \mathrm{DB}, \mathrm{MP}$ 


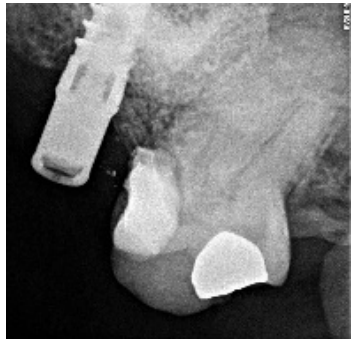

(a)

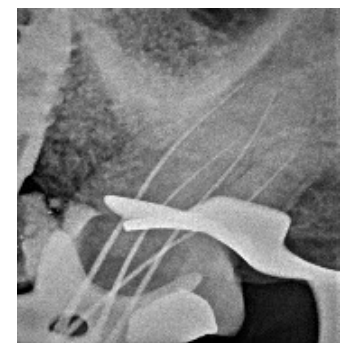

(c)

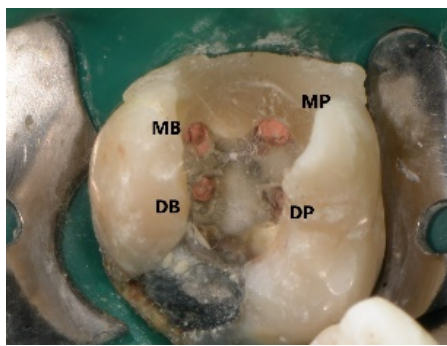

(e)

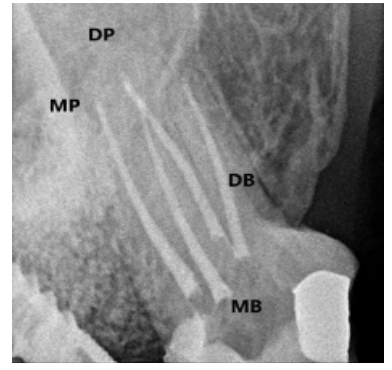

(g)

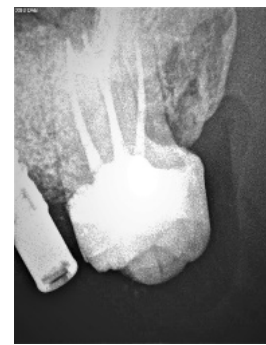

(i)

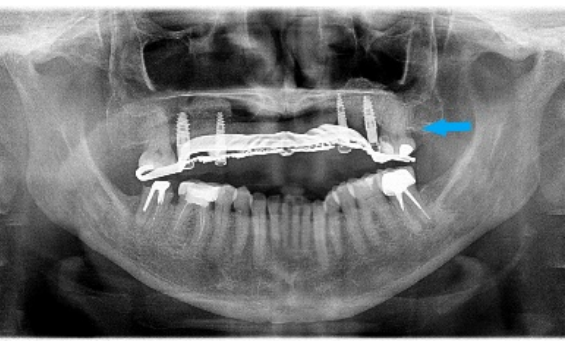

(b)

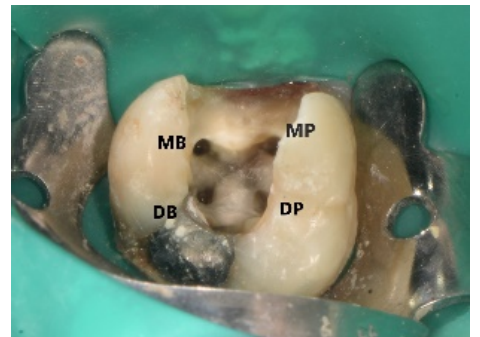

(d)

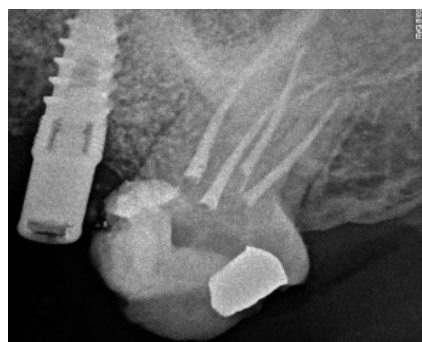

(f)

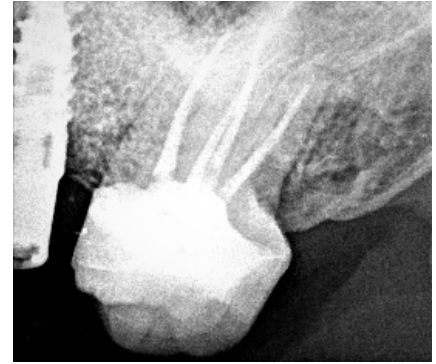

(h)

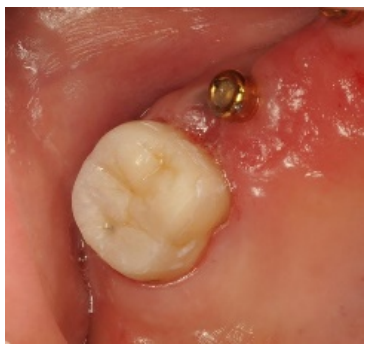

(j)

Figure 1. (a) Pre-op radiograph of maxillary left second molar; (b) Panoramic dental radiography; (c) Working length of the four canals; (d) Clinical view of the pulp chamber with four orifices; (e) Clinical view of the four canals filled with gutta-percha; (f) Immediate post-op radiograph; (g) Immediate post-op radiograph with different view; (h) One year follow-up radiograph showing no radiolucency on the surrounding tissues and final 
restoration; (i) One year follow-up radiograph with different angulation; (j) Clinical view of final restoration after one year

and DP) were instrumented with hand files; SX, S1, S2 ProTaper Gold rotary files (Dentsply Sirona, Charlotte, NC, USA) and 30.04 Vortex Blue rotary file (Dentsply Sirona, Charlotte, NC, USA) as final instrumentation file. The canals were rinsed rigorously with 15\% EDTA solution for 3-5 minutes and dried with size 30 sterile paper points (Figure 1(d)). Obturation was performed using gutta-percha points size 30.04 (Meta Biomed, Chungcheongbuk-do, South Korea) and ADSEAL (Meta Biomed, Chungcheongbuk-do, South Korea) root canal sealer (Figure 1(e)). A sterilized cotton pellet was placed in the pulp chamber, the access cavity was temporarily sealed with IRM (Dentsply, São Paulo, Brazil). Two post-op. periapical radiographs were taken from different angulations (Figure 1 (f) and Figure $1(\mathrm{~g})$ ).

After 12 months follow-up, two radiographs with different angulation were taken and showed no periapical radiolucency. Clinical examinations revealed no tenderness to percussion or palpation tests. Tooth was asymptomatic and functional with final restoration placed (Figures $1(\mathrm{~h})$-(j)).

(Figures 2(a)-(c)) Images of CBCT. Coronal, sagittal and axial views showing anatomic disposition of the four roots (Four-leaf clover) and root canal filling of the four canals.

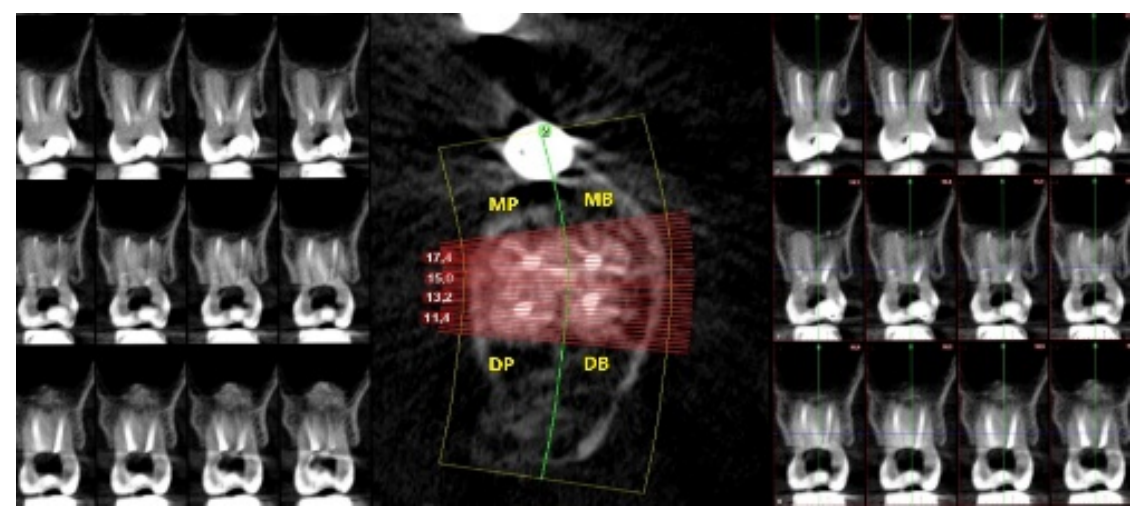

(a)

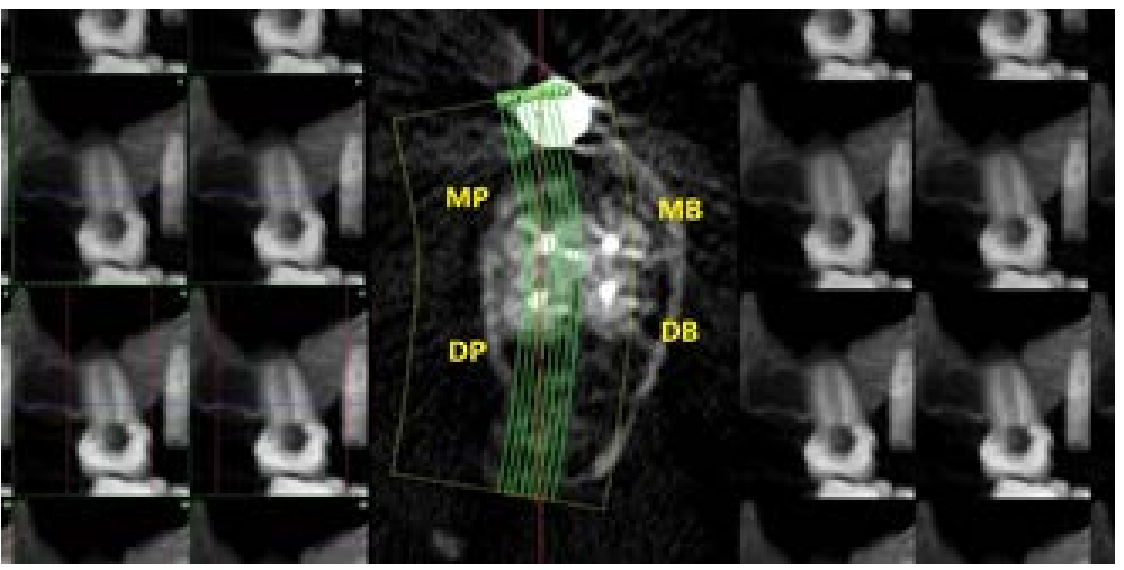

(b) 


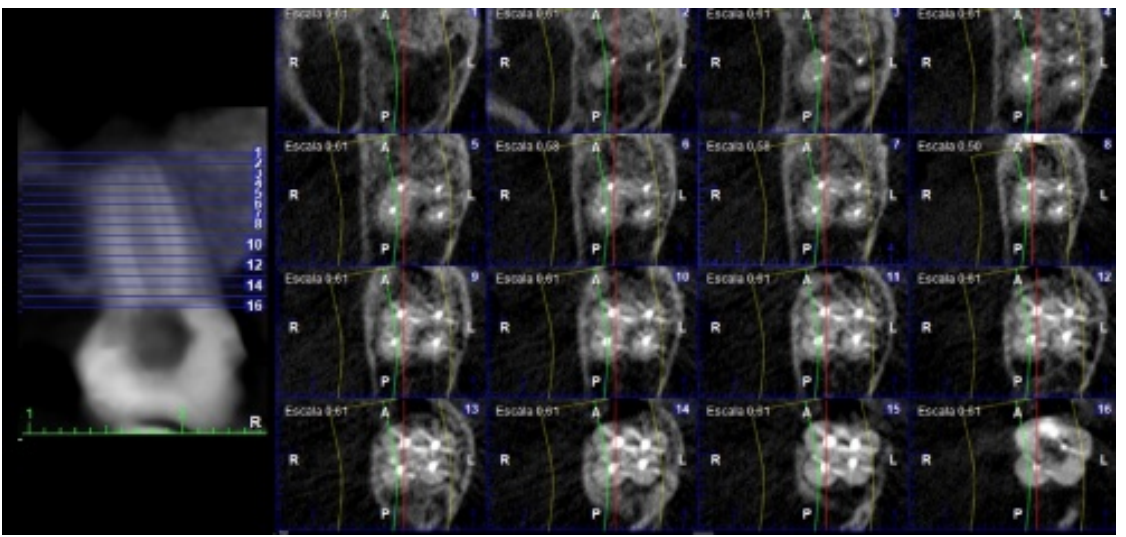

(c)

Figure 2. (a) CBCT post op. sagittal view. Relationship between the four tooth roots and maxillary sinus is evident. (b) CBCT post op. coronal view. Root canal filling at different sections can be observed. (c) CBCT post op. axial views (from apical zone to coronal). Different sections of the four roots/canals and disposition of the four roots/root canal filling at distinct levels can be observed.

\section{Discussion}

The frequency of four-rooted maxillary second molars is scanty in the literature. The complexity of the root canal system of the second maxillary molar is mainly associated to presence of the second mesiobuccal canal. Literature review summarize that this tooth has three roots in its standard anatomical form, variations from this morphology has been considered to be an anomaly. This idea presumes that all maxillary second molars have three roots [5]. The maxillary second molar described in this case, is associated with Type II according to Christie's classification with four separate roots disposed parallel; the roots are short and have blunt apices [9].

A double palatal root is not easy to identify due the superposition of the anatomical structures and zygomatic arch, study of additional shifted radiographs are useful for detecting extra roots. However, periapical radiographs information is insufficient due to the fact that the three-dimensional configuration of the area being radiographed is compressed into a two-dimensional image [10] [11]. In the present case report, the two palatal roots were not detected from the preoperative radiograph. Only after endodontic access completed and aid of dental operating microscope, we were able to find the two palatal canal orifices, thus magnification could increment detection of additional canals. Cone Beam Computed Tomography (CBCT) technology cooperates with the clinician to have a superior comprehension of root canal anatomy. One of CBCT's considerable benefits is the attenuation in radiation exposure [12]. However, CBCT should be used on a case-by-case basis where periapical radiographies do not provide enough diagnostic details.

The aim of endodontic access is to eliminate the pulp chamber roof completely, orifices will be exposed when the roof has been removed and access is completed. Conservancy of tooth structure must be considered when constitut- 
ing endodontic cavity, this may affect future restoration after root canal treatment. Traditionally, cavity access outline for second maxillary molar is triangular, however, in teeth with two palatal roots the design should be square or trapezoidal shape [13].

The orifices of the root canals are localized at the angles in the floor wall junction. Use of ultrasonic tips, coloring the chamber floor with $1 \%$ methylene blue dye and "champagne bubble" tests using sodium hypochlorite are useful for detecting canal orifices [14]. However, it is not unusual for clinicians to miss a root canal. If endodontic files are confined not equidistant in the canal clinically or radiographically, clinician should be suspicious of extra canals.

In this specific case, endodontic cavity shape was quadrangular and canal orifices were equidistant. All root canals were instrumented with hand files and nickel-titanium rotary instruments. Although the advantages of using rotary instruments for cleaning and shaping are well known, most of endodontic procedures still require an integrated use of hand instruments and engine-driven instruments [15].

\section{Conclusion}

The frequency of occurrence of four-rooted maxillary second molars is reduced, however clinician should be aware of the presence of extra root canals. Careful inspection of the pulp chamber, use of magnification and careful observation of radiographs and CBCT images are essential for diagnosis and successful endodontic treatment.

\section{Conflicts of Interest}

The authors declare no conflicts of interest regarding the publication of this paper.

\section{References}

[1] Siqueira Jr., J.F. and Rôças, I.N. (2008) Clinical Implications and Microbiology of Bacterial Persistence after Treatment Procedures. Journal of Endodontics, 34, 1291 301. https://doi.org/10.1016/j.joen.2008.07.028

[2] Gutmann, J.L., Kuttler, S. and Niemczyk, S.P. (2010) Root Canal Obturation: An Update. Academy of General Dentistry, 1.

[3] Nayak, G., Aeran, H. and Singh, I. (2016) Radix Mesiolingualis and Radix Distolingualis: A Case Report of a Tooth with an Unusual Morphology. Restorative Dentistry \& Endodontics, 41, 322-331. https://doi.org/10.5395/rde.2016.41.4.322

[4] Karale, R., Chikkamallaiah, C., Hegde, J., Aswathanarayana, S., Santhosh, L., Bashetty, K., Susheela, S.R. and Panchajanya, S. (2013) The Prevalence of Bilateral Three-Rooted Mandibular First Molar in Indian Population. Iranian Endodontic Journal, 8, 99.

[5] Peikoff, M.D., Christie, W.H. and Fogel, H.M. (1996) The Maxillary Second Molar: Variations in the Number of Roots and Canals. International Endodontic Journal, 29, 365-369. https://doi.org/10.1111/j.1365-2591.1996.tb01399.x

[6] Libfeld, H. and Rotstein, I. (1989) Incidence of Four-Rooted Maxillary Second Mo- 
lars: Literature Review and Radiographic Survey of 1,200 Teeth. Journal of Endodontics, 15, 129-131. https://doi.org/10.1016/S0099-2399(89)80134-7

[7] Alani, A.H. (2003) Endodontic Treatment of Bilaterally Occurring 4-Rooted Maxillary Second Molars: Case Report. Journal-Canadian Dental Association, 69, 733 736.

[8] Shin, S.J., Park, J.W., Lee, J.K. and Hwang, S.W. (2007) Unusual Root Canal Anatomy in Maxillary Second Molars: Two Case Reports. Oral Surgery, Oral Medicine, Oral Pathology, Oral Radiology, and Endodontology, 104, e61-e65. https://doi.org/10.1016/j.tripleo.2007.07.014

[9] Christie, W.H., Peikoff, M.D. and Fogel, H.M. (1991) Maxillary Molars with Two Palatal Roots: A Retrospective Clinical Study. Journal of Endodontics, 17, 80-84. https://doi.org/10.1016/S0099-2399(06)81613-4

[10] Patel, S., Dawood, A., Whaites, E. and Pitt Ford, T. (2009) New Dimensions in Endodontic Imaging: Part 1. Conventional and Alternative Radiographic Systems. International Endodontic Journal, 42, 447-462. https://doi.org/10.1111/j.1365-2591.2008.01530.x

[11] Robledo, J.G. and Fernandez, J.J. (2018) Internal Inflammatory Root Resorption. Management with Mineral Trioxide Aggregate and Gutta-Percha. Journal of Dentistry and Oral Care Medicine, 4, 105. https://doi.org/10.15744/2454-3276.4.105

[12] Cotton, T.P., Geisler, T.M., Holden, D.T., Schwartz, S.A. and Schindler, W.G. (2007) Endodontic Applications of Cone-Beam Volumetric Tomography. Journal of Endodontics, 33, 1121-1132. https://doi.org/10.1016/j.joen.2007.06.011

[13] Rajalbandi, S., Shingte, S.N., Sundaresh, K.J. and Mallikarjuna, R. (2013) Aberration in the Palatal Root of the Maxillary First Molar. Case Reports, bcr2013008641. https://doi.org/10.1136/bcr-2013-008641

[14] Vertucci, F.J. (2005) Root Canal Morphology and Its Relationship to Endodontic Procedures. Endodontic Topics, 10, 3-29. https://doi.org/10.1111/j.1601-1546.2005.00129.x

[15] Shen, L.C. and Gutmann, J.L. (2016) Management of a Mandibular Second Molar with Dilacerated and S-Shaped Root Canals. ENDO, 10, 225-229. 